\title{
Idiopathic Carotidynia: A Cause of Cervical Pain
}

\author{
Vipan Magotra ${ }^{1}$, Monica Manhas ${ }^{2}$, Amit Manhas ${ }^{3}$, Shallu Jamwal ${ }^{4}$, Parmod Kalsotra ${ }^{5}$, \\ Sachin Gupta ${ }^{6}$, Rajiv Kumar Gupta ${ }^{7}$
}

\author{
${ }^{1}$ Associate Professor, Department of Radiodiagnosis, \\ ${ }^{2}$ Assistant Professor, Physiology, \\ 3Lecturer, Department of Anaesthesia, \\ 5Professor, ENT Department, \\ 7Professor, PSM, \\ Govt. Medical College, Jammu, India. \\ ${ }^{4}$ Assistant Surgeon, Health Services, Jammu, India. \\ ${ }^{6}$ Assistant Professor, ENT Department, ASCOMS, Jammu, India
}

\begin{abstract}
Introduction: Carotidynia is a radiating neck pain syndrome associated with tenderness over the carotid bifurcation, without apparent structured abnormality of the carotid artery itself. Due to its controversial aspects, the Headache Classification Subcommittee of the International Headache Society agreed to remove carotidynia as a distinct entity in the $2^{\text {nd }}$ Edition of Classification of Headache Disorders, 2004.

Material and methods: 10 out-patients suffering from idiopathic carotidynia with clinically elicitable "Fay sign" were enrolled and after a detailed clinical examination, their biochemical and radiological investigations were carried out.

Results: A combination of NSAID, oral steroids and antacid therapy showed positive results in five patients out of enrolled ten within one week. The rest five patients, became symptom free with the addition of pregabalin. Two patients had a relapse after 3 months and were managed with a combination of NSAIDs and pregabalin.
\end{abstract}

Conclusion: Idiopathic carotidynia responds to symptomatic

\section{INTRODUCTION}

Carotidynia was first described by Temple Fay in $1927^{1}$ as a symptom of unilateral vascular neck pain. The incidence and prevalence of carotidynia are unknown. Most authors agree that it is common but frequently unrecognized. It may be 2 or 3 times as common as cluster headaches. ${ }^{2}$ In carotidynia, the pain is most often unilateral and localized to the neck, although radiation to the face, ear or molar region is not uncommon and may occur as permanent or periodic pain. Total head movement, swallowing or chewing may aggravate the pain. ${ }^{3}$ Disorders of the autonomic nervous system have never been observed in carotidynia. ${ }^{3}$ In 1988, the International Headache Society ${ }^{4}$ defined the syndrome as shown in Table 1. However, some authors believed carotidynia to be a clinicopathological entity, suggesting that it is merely a symptom of various and heterogeneous causes of neck pain. These controversial aspects led the Headache Classification treatment involving NSAIDs, oral steroids and pregabalin. Patients need to be investigated to exclude any neurological and/or vascular cause.

Key Words: Carotidynia, Neck pain, "Fay sign".

\section{*Correspondence to:}

Dr. Monica Manhas,

Assistant Professor, Physiology,

Govt. Medical College, Jammu, India.

Article History:

Received: 28-09-2016, Revised: 10-10-2016, Accepted: 21-10-2016

\begin{tabular}{|l|c|}
\hline \multicolumn{2}{|c|}{ Access this article online } \\
\hline $\begin{array}{l}\text { Website: } \\
\text { www.ijmrp.com }\end{array}$ & Quick Response code \\
\hline DOI: & \\
10.21276/ijmrp.2016.2.6.016 & \\
\hline
\end{tabular}

Subcommittee of IHS Classification to describe it as distinct entity from the main classification in 2004. ${ }^{5}$ Carotidynia can occur at almost any age. The youngest patient reported in the literature was 10 years old and the oldest 75 years old. Most of the patients were in the $5^{\text {th }}$ decade of life and women were described as being more frequently affected than men. ${ }^{3,6}$ We report our series of 10 patients of idiopathic carotidynia, followed by the diagnosis, possible causes and various treatment modalities for this syndrome.

\section{MATERIALS AND METHODS}

The present study was conducted on outpatient basis in the Department of ENT, Government Medical College, Jammu, over a period of one year and included 10 patients of idiopathic carotidynia. 
Table 1: International Hedache Society Classifcation Committee for the diagnosis of Idiopathic Carotidynia 1988

A. Presence of at least one of the following symptoms overlying the carotid artery:

(1) Painful when applied pressure.

(2) Swelling.

(3) Increased pulsation.

B. Structural lesion in the carotid artery ruled out by means of suitable procedure.

C. Neuralgia of the neck and head with spontaneous improvement within 14 days.

D. Pain on one side of the neck and in the nape, possibly radiating to the head.

Inclusion criteria: Patients with complain of upper cervical pain with 'Fay' sign elicitable and wherein all known causes of cervical pain were excluded.

Exclusion criteria: Patients with following diseases were excluded from the study:

* Cervical lymphadenitis, cervical neoplasms, stylalgia.

* Known cause of cervical pain like thyroiditis, dentogenic pain, temporo-mandibular joint arthritis, temporal arteritis.

* Vascular causes of cervical pain like chronic paroxysmal hemicrania, chronic migrainous neuralgia, cluster headache variant, pericarotid syndrome, trigemmial neuralgia.

* Presence of any neurological and/or any autonomic sign/symptom including Horner's syndrome.

* Radiologically proven carotid artery aneurysm, thrombosis, carotid atherosclerosis.

All patients underwent thorough general physical, ENT and neurological examinations. Carotid bruit was looked for. Blood investigations included complete hemogram, renal and liver function tests, thyroid profile and lipid profile. Radiological investigations included plain X-ray chest, Ultrasonography and CECT neck was done which included cervical spine, styloid process, thyroid gland, submandibular gland and carotid artery.

\section{RESULTS}

The present study included 10 patients of carotidynia (7 females and 3 males), age ranging from 30 to 72 years. All patients had a constant dull pain on one side of the neck, predominantly at the angle of the jaw, with irradiation into the face, ear, eye. One patient had radiation to left temple, one to left temporoparietal area. All patients had throbbing or stabbing exacerbations of pain for different duration of time (half hour to maximum four hours) at varying intervals. Neck movement or swelling was the aggravating factors in few patients. None of the patients had redness of the face, conjunctival injection, lacrimation, nasal congestion, visual symptoms, nausea or vomiting. One patient had in addition a history of migraine unconnected with the cervical pain. Three patients had history of upper respiratory tract infection proceeded to onset of cervical pain. Three patients had cancer phobia.

Table 2: Clinical profile of patients of Carotidynia

\begin{tabular}{|c|c|c|c|c|}
\hline $\begin{array}{l}\text { Case } \\
\text { No. }\end{array}$ & $\begin{array}{l}\text { Sex/Age } \\
\text { (in years) }\end{array}$ & Pain & Physical findings & Other findings \\
\hline 1. & Female/42 & $\begin{array}{l}\text { Dull pain at right of mandible. Throbbing for half an hour after } \\
\text { 3-4 days. Irradiation to right ear. }\end{array}$ & $\begin{array}{c}\text { Tender carotid } \\
\text { bifurcation/ bulb. }\end{array}$ & H/O URTI \\
\hline 2. & Female/30 & $\begin{array}{l}\text { Dull pain at right side of neck. Stabbing for } 30-40 \text { minutes } \\
\text { daily. }\end{array}$ & $\begin{array}{l}\text { Right carotid bulb } \\
\text { tender. }\end{array}$ & H/o URTI \\
\hline 3. & Female/54 & $\begin{array}{l}\text { Dull pressure on right side of neck. Pulling for days, throbbing } \\
\text { for half an hour every day. Irradiation to right ear/eye. }\end{array}$ & $\begin{array}{l}\text { Right carotid } \\
\text { bifurcation tender. }\end{array}$ & H/o migraine \\
\hline 4. & Male/57 & $\begin{array}{l}\text { Dull pain on left side of neck. Stabbing for } 30-40 \text { minutes daily. } \\
\text { Irradiation to left temple and face. }\end{array}$ & $\begin{array}{l}\text { Tender left carotid } \\
\text { bifurcation. }\end{array}$ & $\begin{array}{l}\text { TSH slightly } \\
\text { raised }\end{array}$ \\
\hline 5. & Male/72 & $\begin{array}{l}\text { Dull pain on left side of neck. Throbbing for } 1 \text { to } 4 \text { hours daily, } \\
\text { radiation to left face, temporoparietal area. }\end{array}$ & $\begin{array}{l}\text { Tender left carotid } \\
\text { bifurcation and left } \\
\text { facial artery. }\end{array}$ & Nil \\
\hline 6. & Female/40 & $\begin{array}{l}\text { Dull pain on right side of neck. Throbbing for } 30 \text { to } 45 \text { minutes, } \\
\text { alternate days, no radiation. }\end{array}$ & $\begin{array}{l}\text { Tenderness of right } \\
\text { carotid bifurcation. }\end{array}$ & $\begin{array}{l}\text { TSH marginally } \\
\text { raised }\end{array}$ \\
\hline 7. & Male/47 & $\begin{array}{l}\text { Dull pressure on right side of neck. Stabbing for half an hour } \\
\text { daily. }\end{array}$ & $\begin{array}{l}\text { Tenderness of right } \\
\text { carotid bifurcation. }\end{array}$ & H/O URTI \\
\hline 8. & Female/36 & $\begin{array}{l}\text { Dull pain behind right angle of jaw. Throbbing for } \\
\text { approximately one hour twice a week. }\end{array}$ & $\begin{array}{l}\text { Tenderness of left } \\
\text { carotid bifurcation. }\end{array}$ & Nil \\
\hline 9. & Female/67 & $\begin{array}{l}\text { Dull pain on left side of neck. Irradiation to left eye, ear, } \\
\text { temple, face. Throbbing for half an hour three times a week for } \\
\text { several weeks. }\end{array}$ & $\begin{array}{l}\text { Tenderness of left } \\
\text { carotid bulb }\end{array}$ & Nil \\
\hline 10. & Female/39 & $\begin{array}{l}\text { Dull pain on left side of neck. Throbbing for } 1 \text { to } 2 \text { hour every } \\
\text { day. Radiation to left ear. }\end{array}$ & $\begin{array}{l}\text { Tenderness of left } \\
\text { carotid bulb. }\end{array}$ & Nil \\
\hline
\end{tabular}


Clinically, all the patients had tenderness of the carotid artery bifurcation on palpation and 'Fay' sign was positive ('Fay sign' is elicited if the thumbs are placed on the common carotid artery just below the bifurcation, and the structures pressed back against the transverse cervical processes with a rolling movement, a severe reaction of pain is produced on the side of the atypical neuralgia). ${ }^{2}$ None of the patients had Horner's syndrome. Sweating of the face and neck was symmetrical and normal in all patients. Rest of the general physical examination, ENT examination and cervical nerve function was normal. Blood test including hemogram, LFT and RFT were normal. Two patients had marginally raised TSH. CECT of neck and Doppler sonography of both carotid arteries was normal in all the patients.

Treatment of patients included NSAIDs, oral steroids, short course of antibiotics and antacid. Five patients responded well within one week. In rest five patients, 'pregabalin' was added along with NSAIDs and patients responded well. All patients were symptom free at 3 months follow-up.

After three months follow-up, two patients had relapse (they had responded well initially with combination of antibiotics, analgesics) and were successfully managed with NSAIDs and 'pregabalin'. At 6 months follow-up, all patients were symptom free. None of the patients required anti-depressant treatment.

\section{DISCUSSION}

Carotidynia is a symptom based on a characteristic complaint, accompanied by a unique physical finding. The factors that may precipitate an attack of carotidynia once the syndrome has developed are those that precipitate an attack of migraine ${ }^{7}$ : stress, the 'letdown' following stress, certain dietary factors, bright lights, loud sounds, fasting and menstruation. An episode of carotidynia may last for hours or week, but the usual duration is 7 to 10 days. Symptoms may wax and wane over many months without disappearing. After a symptom-free interval of weeks or months, another episode may occur. ${ }^{8}$

The pain of carotidynia is most often unilateral and localized to the neck, although radiation to the face, ear or molar region is not uncommon. It is frequently described as dull and throbbing in character and is continuously present, although day-to-day or hour-to-hour exacerbations and remissions are common. Severity varies from mild to agonizing and the pain is frequently aggravates by swallowing, yawning, coughing, sneezing or elevating the head while moving it towards the contralateral side. ${ }^{2} \mathrm{~A}$ local process involving the carotid artery appears to cause local pain and tenderness as well as referral pain to the mandible, face, eyes, ears and head. The pain is produced by stimulation of the nerve endings of the carotid plexus as described by Fay as a neurogenic cause. Fay, in his study, deducted that vagus nerve and the cervicothoracic spinal nerve roots were the neurological pathway. ${ }^{1}$ In 1948, Wolff ${ }^{9}$ commented that carotidynia was due to vascular change and in the next year, Hilger ${ }^{10}$ ascribed it as an autonomic defect causing carotid artery vasodilation. Lovshin ${ }^{11}$ described the clinical picture of carotidynia in his study of 100 patients and pointed out that many of the patients had vascular symptoms and the same personality traits as migraine patients. Many of the patients had their pains on weekends. Roseman ${ }^{12,13}$ also noted the similarity of carotidynia and migraine. It has been noted that an increase in carotidynia symptoms occur in cold weather and a viral origin of this condition has been suggested. ${ }^{14-16}$
Reported causes of carotidynia include migraine, viral or past infection, giant cell (temporal) arteritis, carotid artery aneurysm, total carotid artery occlusion. ${ }^{2}$ Maximum number of times, carotidynia is idiopathic, also known as benign carotidynia. Tardy ${ }^{17}$ have suggested that the 'carotiditis' may be more appropriate to distinguish the idiopathic inflammatory disorder of carotid arteries from alternate causes of cervical pain syndrome. Others have advocated the term carotid periarteritis. ${ }^{18,19}$ O'Neill et al. ${ }^{20}$ ascribed two basic categories of carotidynia - one a migrainous disorder and the other a structural disease of the carotid artery, while Vander Bogt et al. ${ }^{21}$ commented that carotidynia is a diagnosis of exclusion sporadically used by neurologists and ENT specialists for headaches without specific physical substrate.

The clinical features of carotidynia differ clearly from other pain syndromes of the face and neck. 1,3,6,12 While in facial neuralgia pain and irradiation of pain are usually confined to the area of innervations of the involved nerve, pain in carotidynia is mainly located at the carotid bifurcation and irradiates along its branches. In carotidynia, a real triggering of pain has never been found unlike neuralgic pain. Furthermore, carotidynia typically consists of continuous dull pressure like pain with episodic exacerbation of throbbing lasting several hours. ${ }^{3}$ As a pain of vascular origin, carotidynia must be differentiated from chronic pain syndromes like chronic paroxysmal hemicrania, chronic migraine neuralgia, cluster headache variant and the pericarotid syndrome.,322 The essential differentiating factor is absence of disorder of autonomic nervous system. In vast majority of cases reported in literature, no specific cause of carotidynia could be found $33,6,8,10,11,14,23,24$ and several of these patients also suffered from migraine and since they usually responded well to anti-migraine treatment, a vascular dysfunction similar to that in migraine was postulated as the pathogenetic mechanism. 3,6,10,11,23,24,25 Some authors have also described symptomatic form of carotidynia., 315,26

The typical findings on ultrasound examination are mainly hypoechoic wall thickening of the carotid bulb with a mild to moderate lumen narrowing and a large outward extension of the vessel wall without any acceleration of the blood flow. ${ }^{27}$

The presence of abnormally enhancing soft tissue surrounding the carotid bifurcation is a common radiological finding in idiopathic carotidynia on computed tomography and MRI. Soft tissue enhancement surrounding the carotid bifurcation and wall thickening of the distal common carotid artery were reported by CT scan. However, soft tissue enhancement may be weak on CT. ${ }^{19,28}$

Syms et al. ${ }^{29}$ in 1997, first described MR findings in a patient with carotidynia, abnormally enhanced tissue surrounding the symptomatic carotid artery without haemodynamic changes. It has been observed that the structural changes were limited to adventia of the carotid artery. ${ }^{18}$ In the unique histological study of the abnormal perivascular tissue surrounding the carotid, Upton et al. ${ }^{18}$ demonstrated the presence of non-specific inflammatory changes including vascular proliferation, fibroblast proliferation and low-grade chronic active inflammation.

Proper management of carotidynia depends on the underlying cause. Important point in management is eliciting a history of medical conditions, such as migraine or upper respiratory tract infection and other symptoms especially neurological or ischemia. A careful examination including oral and dental examination, neck 
palpation and possibly indirect or fibroptic laryngoscopy, is mandatory. ${ }^{2}$ In presence of neurological signs or symptoms, a duplex colour Doppler and depending on the result as well as the presentation of the patient, either MRI (magnetic resonance imaging) or arteriography should be performed. In most cases, the ESR and neurologic history and examination will be normal and diagnosis of benign carotidynia can be made. Common treatment of carotidynia is the administration of non-steroidal antiinflammatory drug or glucocorticoids due to a suspected inflammation process. ${ }^{27}$

Patients with a history of migraine and prolonged but mild course of carotidynia may be treated with appropriate migraine therapy. Young patients with more acute course, no history of migraine or symptom, following viral infection may be treated symptomatically i.e. reassurance, anti-inflammatory agents and time. Methysergide, ergot preparations, pizotyline and propranolol have been used for migrainous carotidynia. ${ }^{8}$ Indomethacin or flurbiprofen is also advocated for treatment of carotidynia. ${ }^{3}$ The ultimate treatment in severe and recalcitrant cases may be denervation of the carotid bulb, also recommended by Fay, ${ }^{1,2}$ which is radical approach and should be undertaken in rare cases with no other opinion. ${ }^{2}$

\section{CONCLUSION}

Benign or idiopathic carotidynia is usually a self-limiting condition presenting with cervical pain not attributable to any known cause and may be treated symptomatically. Patient should be investigated properly to exclude any neurological and/or vascular pathology of carotid as underlying cause presenting clinically as carotidynia.

\section{REFERENCES}

1. Fay T. Atypical neuralgia. Arch Neural Psychiatry 1927; 18: 309-15.

2. Hill LM, Hasting G. Carotidynia: a pain syndrome. J Fam Pract 1994; 39: 71-5.

3. Orfei R, Meienberg O. Carotidynia: report of eight cases and prospective evaluation of therapy. J Neurol 1983; 230: 65-72.

4. Headache Classification Committee of the International Headache Society. Classification and diagnostic criteria for headache disorders, cranial neuralgia and facial pain. Cephalalgia 1988; 8 (Suppl 7): 1-96.

5. Headache Classification Committee of the International Headache Society. The International Classifiation of Headache Disorders, 2nd edition. Cephalgia 2004; 24(Suppl 1): 9-160.

6. Lovshin LL. Carotidynia. Headache 1977; 17: 192-5.

7. Murray TJ. Migraine. NS Med Bull 1977; 56: 15.

8. Murray TJ. Carotidynia: a cause of neck and face pain. CMA J 1979; 120: 441-3.

9. Wolff HG. Headache and other head pain. Oxford U Pr New York 1948: 642.

10. Hilger JA. Carotid pain. Laryngoscope 1949; 59: 829-38.

11. Lovshin LL. Vascular neck pain - a common syndrome seldom recognized. Cleve Clin Q 1960; 27: 5-13.

12. Roseman DM. Carotidynia - a distict syndrome. Arch Otolarngol 1967; 85: 81-4.

13. Roseman DM. Carotidynia, headaches and cranial neuralgia.
In: Vinkan PG, Bruyn GW (editors), Handbook of Clinical Neurology, Vol. 5. Elseveir - North Holland, Amsterdam, 1968: pp. $375-8$

14. Saunders WH. Carotid arteritis, a new treatment for an often overlook condition. Laryngoscope 1962; 72: 481.

15. Roseman DM. Carotidynia - a distict syndrome (E). JAMA 1967; 199(2): 121.

16. Comacchio F, Bottin R, Brescia G, Tsilikos K, Volo T, Tregnaghi $A$, et al. Carotidynia: new aspects of a controversial entity. Acta Otorhino Laryngol Italica 2012; 32: 266-9.

17. Tardy J, Pariente J, Nasr N, et al. Carotidynia: a new case for an old controversy. Eur J Neurol 2007; 14: 704-5.

18. Upton PD, Walker Smith JG, Charnock DR. Histologic confirmation of carotidynia. Otolaryngol Head Neck Surg 2003; 129: 443-4.

19. Hafner F, Hackel G, Haas E, Ellar P, Gstettner C, Vollmann R, et al. Idiopathic carotidynia. Vasa 2014; 43: 287-92.

20. O'Neill B, Aronson A, Pearson B, Nauss L. Superior laryngeal neuralgia: carotidynia or just another pain in the neck. Headache 1982; 22: 6-9.

21. Vander Bogt KEA, Palm WM, Hamming JF. Carotidynia: a rare diagnosis in vascular surgery practice. E Jves Extra 2012; 23: e18-e19.

22. Vijayan N, Watson C. Pericarotid syndrome. Headache 1978; 18: 244-54.

23. Davies JVSA. Carotid pain: report of six cases. Br Med J 1961; 2: 1528-9.

24. Ford FR. The carotid pain syndrome. Report of two cases which suggest that in some instances migraine is responsible. Bull Johns Hopkins Hosp 1964; 114: 266-8.

25. Martorell F. Carotidynia. Acta-Oto-Rhino-Laryngol-Ibero-Am 1961; 12: 116-20.

26. Roseman DM. Carotidynia. NY State J Med 1963; 63: 2651-3.

27. Schaumberg J, Eckart B, Michel P. Carotidynia. Magnetic resonance imaging and ultrasonographic imaging of a self-limiting disease. Clin Neuroradiol 2011; 21(2): 91-4.

28. Park JK, Choi JC, Kims BS, Choi G, Kim SK. CT imaging features of carotidynia: a case report. J Neuroimaging 2009; 19(1): 84-5.

29. Syms MJ, Burton BS, Burgess LPA. Magnetic resonance imaging in carotidynia. Otolaryngol Head Neck Surg 1997; 117: 156-9.

Source of Support: Nil. Conflict of Interest: None Declared. Copyright: (c) the author(s) and publisher. IJMRP is an official publication of $\mathrm{lbn}$ Sina Academy of Medieval Medicine \& Sciences, registered in 2001 under Indian Trusts Act, 1882.

This is an open access article distributed under the terms of the Creative Commons Attribution Non-commercial License, which permits unrestricted non-commercial use, distribution, and reproduction in any medium, provided the original work is properly cited.

Cite this article as: Vipan Magotra, Monica Manhas, Amit Manhas, Shallu Jamwal, Parmod Kalsotra, Sachin Gupta, Rajiv Kumar Gupta. Idiopathic Carotidynia: A Cause of Cervical Pain. Int J Med Res Prof. 2016; 2(6):88-91.

DOI: 10.21276/ijmrp.2016.2.6.016 